\title{
Energy control by linking individual patterns to self-repeating diffractive optical elements
}

\author{
C. Y. Lu, H. Z. Liao, C. K. Lee, and J. S. Wang
}

\begin{abstract}
In general, as diffractive optical elements formed by use of self-repeating patterns possess beneficial characteristics such as scratch resistance, low design effort, ease of fabrication, and natural formation of large panels, an efficient design methodology that was developed with a modified preserving-the-best strategy of genetic algorithms is presented. Both genetic algorithms and simulated annealing are examined by the Markov-chain stochastic process to create the insight needed to use these two heuristic algorithms efficiently. It was found that adding the preserving-the-best strategy to traditional genetic algorithms guarantees the possibility of locating the global optimum. Combining this sufficient and necessary condition for locating a global optimum for genetic algorithms with the built-in chromosome crossover searching mechanism and its neighborhood identification makes this newly developed genetic algorithm an effective method for designing diffractive optical elements. In our study, a prototype was fabricated based on our case study with the modified genetic algorithm. The performance of this prototype was measured and analyzed. Experimental results are shown to agree well with theoretical predictions. (C) 1997 Optical Society of America
\end{abstract}

Key words: Diffractive optical elements, binary optics, genetic algorithms.

\section{Introduction}

Using self-repeating patterns to form large diffractive optical elements (DOE's) provides us with a way to create a DOE that is virtually scratch resistant, similar to that of traditional holograms. More specifically, self-repeating patterns can be used to store information that will not be damaged even when surface patterns are partially damaged. Using this concept to generate large-format DOE's has been shown to reduce significantly computational time and other costs involved because of its fundamental characteristics. DOE's generated by the combination of selfrepeating patterns are thus examined in this paper to investigate the possibility of distributing input light beams into user-specified energy patterns; that is, one of our main objectives in this paper is to examine the methodologies of designing DOE's capable of assigning prespecified output-energy distribution ratios to any user-defined direction.

Currently there are many computational algorithms that exist for designing DOE's. ${ }^{1-3}$ However,

The authors are with the Institute of Applied Mechanics, National Taiwan University, Taipei, Taiwan, China.

Received 4 September 1996; revised manuscript received 19 December 1996.

0003-6935/97/204702-11 $\$ 10.00 / 0$

(C) 1997 Optical Society of America the design of complex DOE's is typically viewed as a search for solutions to minimization problems in multidimensional discrete variable domains. Iterative algorithms, ${ }^{4-8}$ which can be classified into bidirectional iterative algorithms, such as the iterative Fourier-transform algorithms ${ }^{9-13}$ (IFTA), and the unidirectional iterative algorithms, such as the heuristic algorithms, ${ }^{14-20}$ are typically used in approaching these types of problems. The main difference between the bidirectional and the unidirectional algorithms lies in the requirement of identifying the inverse mapping within the algorithm. More specifically, using a bidirectional iterative algorithm requires a fundamental understanding of not only the influence that the DOE has on the image produced and on the design metric, but also of how variations in the response affect the DOE.

As only scalar-domain analysis is discussed in this paper, either a Fresnel diffraction or a Fourier diffraction is quite applicable in the design of DOE's examined here. Thus algorithms such as the IFTA can be readily applicable for these types of problems. However, it should be noted that, even though the IFTA is simple to implement, it is prone to stagnate in local minima. ${ }^{1,11,12}$ Exploitation of design freedom to redistribute DOE design values while securing the desired performance objective in the image plane is a necessary condition to adopting the IFTA effectively. On the other hand, a unidirectional al- 
gorithm characterizes DOE's by a finite set of quantified parameters and then executes a finite but typically large number of permutations of these design parameters. For the case in which the model of the optical systems cannot be easily inverted, a unidirectional algorithm should be the choice. Although both types of algorithms can be successfully adopted in designing the DOE's of this paper, our efforts are concentrated on revealing the fundamental mathematical structures of the most popular unidirectional algorithms, i.e., the genetic algorithm ${ }^{14-17}$ (GA) and simulated annealing ${ }^{18-20}$ (SA), in order to facilitate the use of these algorithms.

In an attempt to optimize the numerical coding process, a stochastic process is adopted in this paper to examine the fundamental relationship between the GA and SA. Furthermore, the understanding obtained from this paper provides us with an opportunity to adjust the tuning parameters that exist within these algorithms more intelligently. It is also identified that a modified GA with a preservingthe-best strategy has the necessary and sufficient conditions to ensure the convergence of the numerical calculations. Even though SA is not examined in great detail in this paper, the stochastic process we have adopted has been shown to provide us with a way to code these two important heuristic algorithms in an identical manner with some minor modifications.

A $16 \times 16$ pixel array was designed as a unit cell for the repetition of the large DOE panel in order to redistribute the incident light beam into a desired pattern. As the first three positive and negative orders of diffractive light were considered for the output pattern, a total of $7 \times 7$ points were specified. With our design approach, the ability to specify arbitrarily the energy ratio within each of these $7 \times 7$ points produces a significant amount of information that can be stored in such a simple pattern. To examine the sensitivity of our final design, a $64 \times 64$ pixel array of a self-repeating unit with respect to a $9 \times 9$ output point was also calculated to compare results.

The design patterns were generated with a commercially available imagesetter, ${ }^{21,22}$ such as the Agfa SuperSelect 7000 reticle mask. The binary patterns generated by the imagesetter can be transferred into a reticle through a photoreduction process. ${ }^{23}$ The reticle is then used to transfer the intended pattern onto a photoresist by direct-contact photolithography. A phase-type DOE can then be fabricated by either a wet-etch or a dry-etch technique after the photoresist is developed.

The surface relief generated by the etching process serves as a phase variation to generate the DOE prototype of choice. A binary DOE with the selfrepeating elements mentioned above was fabricated with this process. Experimental results were compared with the theoretical predictions in order to examine the efficiency and accuracy of our newly developed approach.

\section{Theory}

Even though the rigorous coupled-wave theory has been so well examined ${ }^{24-30}$ in the past, the scalar diffraction theory still holds a place when the feature size of the DOE is not too close to that of the wavelength. ${ }^{31}$ Many constraints exist in identifying a DOE pattern that can transfer an incident light beam into an arbitrarily specified intensity pattern. These constraints include limiting the smallest feature size that can be fabricated and limiting the maximum phase levels achievable during tasks such as multimask alignment. With so many existing constraints, in addition to the large number of discrete variables that need to be examined, a direct combinatorial search for the solutions of such problems usually becomes impractical.

In contrast to the direct approach mentioned above, these types of problems can generally be satisfied with a heuristic search algorithm. SA and GA's are the two popular search methods that have been found to search for the desired solution ${ }^{19,31}$ efficiently. These two algorithms are briefly reviewed here.

\section{A. Simulated Annealing}

In 1953, Metropolis et al. used the Monte Carlo method to simulate a many-atom system. ${ }^{32}$ At an arbitrary temperature $T$, a specific molecular configuration $c$ will have a total energy state $E(c)$. According to Boltzmann's distribution, the probability of this configuration is $\exp [-E(c) / k T]$, where $k$ is the Boltzmann constant and $T$ is the absolute temperature. As this system has a greater probability of residing in the lower-level states, it tends to decline to configurations that have lower energy states. Nonetheless, as this system continues to maintain the probability of jumping from a lower energy state to a higher energy state, the system does not always stick to the lowest configuration. Thus the energy of each individual state determines the probability of jumping between states, and this makes the system more likely to reside in the global-minimum energy state as the temperature decreases eventually to $0^{\circ}$. This type of searching algorithm is thus called ${ }^{18} \mathrm{SA}$, as the common manufacturing procedure is to heat the system to a high temperature and then cool it down slowly in order to improve the structure.

When the SA is applied to configuring an optimization problem, the following steps must be specified in order to simulate a thermodynamic system:

1. Determine all possible configurations as well as the neighborhood of each configuration.

2. Decide on a random transition method.

3 . Define an energy function to evaluate the energy of each configuration and identify the evaluated energy minimum as the optimization goal of the system.

4. Decide the temperature $T$ and the annealing schedule that can attain the goal and that is reasonably efficient; the algorithm can be written in a pseudocode, as shown in Table 1. 
Randomly produce the initial configuration, termed

current_config, and set up $T$

while(not thermal equilibrium)

\{

search the local neighborhood minimum, and see if replacing

current_config is needed.

Transition leads to new_config

Evaluate $\Delta E=E$ (new_config) $-E$ (current_config)

If $(\Delta E<0)$ replace current_config by new_config

if $(\Delta E>=0)$ set the probability of replacing

the current config by a new config $=\exp [-\Delta E / T])$

Cool down to $T$ by annealing schedule

\}

Complete the cool down process, and print out the results.

\section{B. Genetic Algorithm}

The GA was developed by Holland at the University of Michigan. ${ }^{15}$ This algorithm simulates the evolutionary principles in nature. The steps can be explained in biological terms. As shown in Fig. 1, all possible modules are first encoded from codes called chromosomes. Second, the adaptive ability of the chromosomes to the circumstances are defined to evaluate the fitness of each chromosome. Third, a significantly large group of chromosomes is chosen to provide the crossover among each individual chromosome and to add the probability of mutation in the course of producing offspring so as to produce a totally new chromosome. Finally, after several generations, the surviving individual chromosome is the one that is the most adaptable to the existing environment.

The following pseudocode itemizes the objects needed to simulate an optimization problem by mimicking an evolutionary system:

1. Generate groups that are large enough to represent different modules.

2. Define a function to describe the fitness of each individual chromosome to the environment in order to lay out the evolutionary rule.

3. Decide on a mechanism for chromosome mutation.

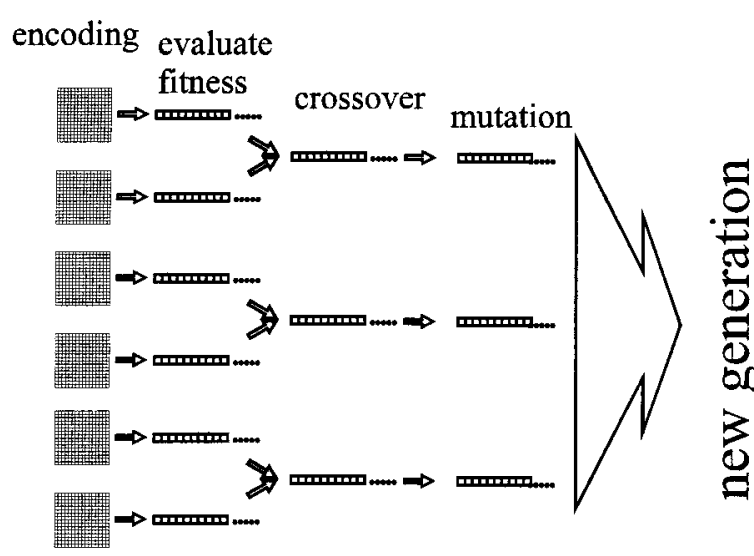

Fig. 1. Sketch of the evolution of each generation in the GA.

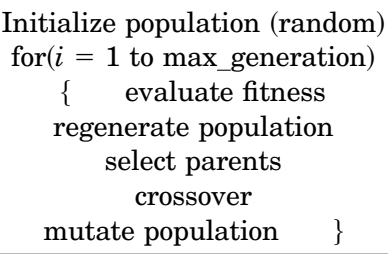

The algorithm is written as shown in Table 2 .

Examining the above-mentioned methods according to the traditional approach makes them appear quite different. Here we show the similarity of these two methods and prove that our newly developed GA with its preserving-the-best strategy possesses the sufficient and necessary condition for convergence. It should be noted that the word convergence is used in a pure mathematical sense, in which convergence means that the final value does not have any possibility of moving away from its current location through evaluation of the merit function, that is, the convergent value corresponds to the global optimum in a heuristic algorithm.

C. Mathematical Structures of the Genetic Algorithm and Simulated Annealing: Markov Chains

Basically the makeups of all SA and GA searches are all Markov chains. ${ }^{33,34}$ We can begin by defining the limited searching space $\Omega$, which in itself is a collection of all possible configurations. The symbol $\mu$ is defined and set to measure the probability of finding solution $X$ in $\Omega$, and it is this probability distribution that we would like to converge on within the searches. For the best result of $x_{0}$, we hope that $\mu\left(x_{0}\right)=1$ and that all other configurations with probability distributions equal zero. Denoting the probability of transition of $y \in \Omega$ to $x \in \Omega$ as $p(y, x)$ and the probability distribution of the $n$th transition as $v_{n}$ yields

$$
v_{n+1}(x)=\sum_{y \in \Omega} v_{n}(y) p(y, x)
$$

As $v_{n}$ approaches $\mu$ when $n$ approaches $\infty$ is the definition of convergence, we have

$$
\mu(x)=\sum_{y \in \Omega} \mu(y) p(y, x)
$$

as an invariant ${ }^{35}$ and a necessary condition for convergence. However, because Eq. (2) is difficult to prove, as we would need to assemble all the possible configurations, we can alternatively use the following reversible condition to guarantee the invariant condition state in Eq. (2), where

$$
\mu(y) p(y, x)=\mu(x) p(x, y) .
$$


The statement in Eq. (3) can easily be proved by the combination of Eq. (3) and the constraint $\Sigma_{y} p(x, y)=$ 1 , yielding

$$
\begin{aligned}
\mu(x) & =\mu(x) \sum_{y} p(y, x)=\sum_{y} \mu(x) p(y, x) \\
& =\sum_{y} \mu(y) p(y, x) .
\end{aligned}
$$

According to Eq. (2), this is exactly the invariant.

It can be easily proved that the Metropolis algorithm used in SA satisfies the reversible condition. ${ }^{36}$ More specifically, by use of $H(x)$ as the merit function, $\#(\Omega)$ as the number of $\Omega, Z$ as the normalization factor for $\Sigma_{y} \mu(y)=1$, and $T=T(n)$ as the temperature in the $n$th transition, it is essentially the same as having a family of probability distributions that satisfies the invariant condition but with a dynamically tuning variable $T$; that is,

$$
\begin{aligned}
& p(x, y)=p_{T}(x, y)=\min \left[1, \mu_{T}(y) / \mu_{T}(x)\right] / \#(\Omega) \\
& \text { for every } y \neq x \text {, } \\
& p(x, x)=p_{T}(x, x)=1-\sum_{y \neq x} p_{T}(x, y), \\
& \mu_{T}(x)=\exp [-H(x) / T] / Z .
\end{aligned}
$$

As $T$ gradually decreases to $0^{\circ}, \mu_{T}$ approaches $\mu$, where

$$
\mu= \begin{cases}1 & \text { for the best configuration } x_{0} \\ 0 & \text { for other configurations }\end{cases}
$$

If the random process converges, then it will converge to the best solution, such as when $T=0$ :

$$
\begin{array}{ll}
p_{0}(x, y)=1, & \text { if } \mu(y)>\mu(x), \\
p_{0}(x, y)=0, & \text { otherwise; }
\end{array}
$$

the process will not have any chance to transit to a worst case. In this case, this process is similar to a common random search.

Another example that satisfies the reversible condition is the Gibbs sampler. ${ }^{36}$ The definition is shown to be

$$
\begin{aligned}
p(x, y)= & \{\mu(y) /[\mu(x)+\mu(y)]\} / \#(\Omega) \\
& \text { for every } y \neq x, \\
p(x, x)= & p_{T}(x, x)=1-\sum_{y \neq x} p_{T}(x, y) .
\end{aligned}
$$

For GA's, if there are $k$ chromosomes within each generation, the search will proceed in the space $\Omega^{k}$, denoted as

$$
X=\left(x_{i 1}, x_{i 2}, x_{i 3}, \ldots, x_{i k}\right) \in \Omega^{k} .
$$

Because GA's strive to select the best group within each generation to pass on to the chromosomes, $X$ will not be unique. Such a group is defined as

$$
M_{x_{i}}=\left\{X \in \Omega^{k} \mid \text { the best element in } X \text { is } x_{i}\right\} .
$$

If the best chromosome is located at $M_{x_{0}}$, the best result $x_{0}$ is obtained. Thus the transition can be considered to proceed within $U=\left\{M_{x_{i}} \mid x_{i} \in W\right\}$. Following the same argument as shown with SA, the invariant is shown to be the necessary condition for convergence, i.e.,

$$
\mu(x)=\sum_{y \in \Omega} \mu(y) p\left(M_{y}, M_{x}\right),
$$

where $p\left(M_{y}, M_{x}\right)$ is the transition probability. In contrast to the Metropolis algorithm for SA and the Gibbs sampler described above, the format and the mathematical structure of $p\left(M_{y}, M_{x}\right)$ for the GA are complex and have not been clearly discussed before.

It is required that, for the GA to converge to $x_{0}$, the condition must exist that $\mu\left(x_{0}\right)=1$ and everything else equals zero. Under this condition, Eq. (15) becomes

$$
\mu(x)=\mu\left(x_{0}\right) p\left(M_{x_{0}}, M_{x}\right) .
$$

If $x \neq x_{0}, \mu(x)=0$ as shown above, then

$$
p\left(M_{x_{0}}, M_{x}\right)=0 .
$$

This is a necessary and required condition for the GA to converge. More specifically, this derivation indicates the probability that the transition from a best case to a worst case must be zero in order to guarantee convergence. Thus preserving the best is a necessary condition for convergence in the GA.

Even though the above derivations suggest only the preservation of $x_{0}$ and not the best of each generation, we in fact do not know which one is the best and thus need to identify it generation by generation. Therefore it is clear that preserving the best within each generation is a necessary condition for convergence. It should be stressed that the convergence mentioned here is used in a rigorous mathematical sense, that is, if the chromosome configuration has any possibility of moving away from its current position, in our case we do not consider it convergent. This is distinctly different from locating the local optimum. More specifically, by convergence we mean locating the global optimum in the GA, which also corresponds to locating the global optimum for SA when $T=0$.

The sufficient conditions for convergence can also be proved easily. As there are only finite mutation probabilities in the GA, the search mechanism within a GA is global. Thus it can also be said that the evolutionary history in $\Omega$ or $U$ is ergodic. Because the space for searching is finite, the probability of missing $x_{0}$ is zero. Thus, given enough generations, the preserving-the-best strategy can accurately locate $x_{0}$ and can possess the sufficient condition for convergence.

If $k=1$ in Eq. (13), then $X$ of Eq. (13) is $x_{1}$, which is identical to the $x$ in Eq. (5) of SA. In addition, the transition possibility $p\left(M_{x}, M_{y}\right)$ shown in Eq. (15) reduces to $p(x, y)$ in Eq. (5). Under this condition, the GA is quite similar to SA. If we adopt an extra parameter $T$ as shown in Eq. (5), the reduced GA will 


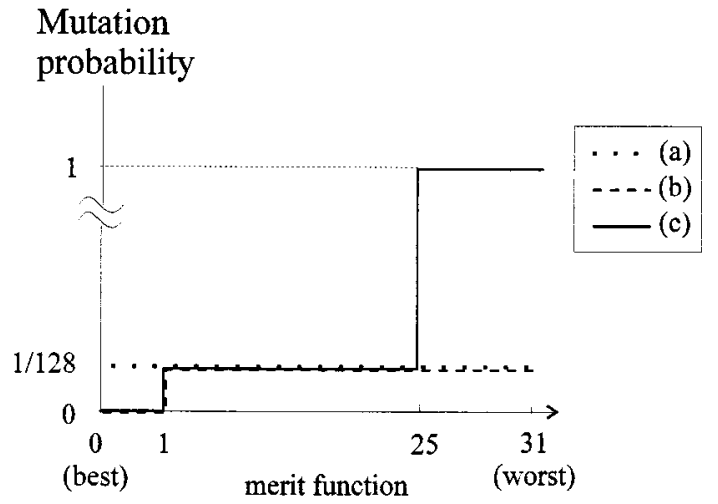

Fig. 2. Graphic representation of the probability distribution of mutation for different GA's: (a) pure GA, (b) modified GA, (c) modified GA with a random-search procedure.

turn into SA. Another thing that should be noted is that the preserving-the-best strategy cannot be adopted in this reduced GA as no progression will be possible. This certainly corresponds well with the common notion that no preserving-the-best strategy is useful or even feasible in SA.

The above discussions clearly spell out that the preserving-the-best strategy can be used to modify the more conventional GA's to guarantee convergence. One thing that should be noted is that, even though the more traditional GA's may identify the best solutions, convergence cannot simply be guaranteed without the adoption of the preserving-the-best strategy. More specifically, introducing the preserving-the-best strategy guarantees the possibility of locating the global optimum. However, to prevent the searching algorithm from locking at a local minimum, a higher possibility of mutation can be introduced into the algorithm to prevent premature termination (Fig. 2). In other words, if the possibility of mutation is not large enough, introducing the preserving-the-best strategy might induce a premature locking at a local optimum. This understanding provides us with an insight of what variables might be useful in ensuring that the global optimum is found, that is, introducing a higher possibility of mutation such as adding a random-search process into the preserving-the-best strategy (Figs. 2 and 3)

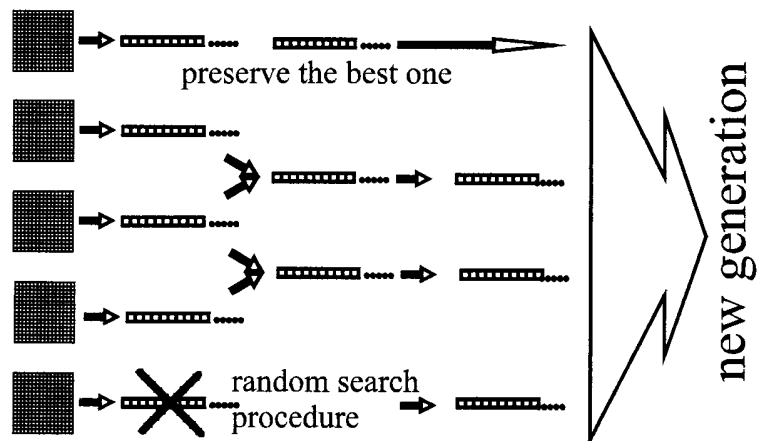

Fig. 3. Modified preserving-the-best strategy GA with a randomsearch procedure.

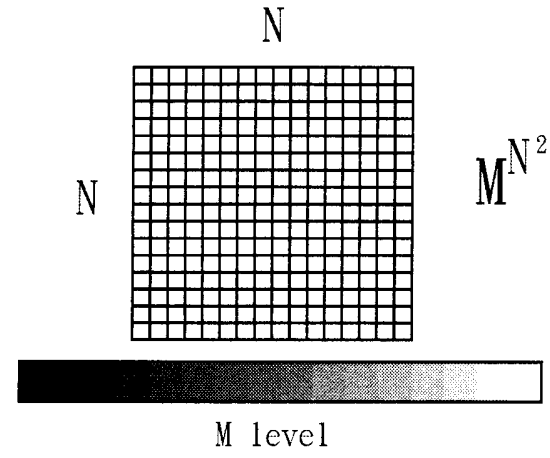

Fig. 4. Individual $N \times N$ unit cell with an $M$-level phase for a self-repeating DOE.

in the GA increases the chances of locating the global optimum. The drawback of this approach is that the iteration cycles will be longer in the beginning. One potential modification might be to increase gradually the possibility of mutation as the GA progresses toward global optimum.

Examining both SA and the GA from the Markovchain viewpoint revealed the following: (1) the invariant condition, which guarantees that the global optimum can be reached, is readily available for SA, but is not clearly defined for the traditional GA; (2) the searching mechanism and the SA neighborhood are not clearly defined, which indicates that if the user cannot identify the most appropriate searching mechanism and neighborhood, SA can be considered almost a random search; (3) the chromosome crossover and population evolution rule for the traditional GA clearly spells out the searching mechanism and neighborhood required; and (4) the preserving-the-best strategy makes the GA algorithm complete, as not only can the global optimum be reached, but also the searching mechanism and the neighborhood are readily available. The above-mentioned results indicate that if the user cannot easily or cleverly identify the searching mechanism and neighborhood of SA or even that of the Gibbs sampler, a GA should be the chosen algorithm. It is because of this understanding that only GA's are applied to the design cases below.

\section{Design Cases}

A two-dimensional (2-D) phase fan-out grating was used as the test case to examine our numerical scheme. A 2-D phase fan-out grating is a combination of two perpendicular one-dimensional gratings, whose structures are periodic in both directions. For the case in which the unit cell of the grating is an $N \times N$ array (Fig. 4) and each pixel of the unit is an element, in addition to $M$ number of phase levels within each element, there will be a total of $N^{2}$ power of $M$ possible situations. The phase level $M$ is limited primarily by its fabrication capabilities. 
If $\otimes$ represents convolutions, the transmittance of the DOE of interest can be written as

$$
\begin{aligned}
t(x, y)= & \sum_{m=-\infty}^{\infty} \sum_{n=-\infty}^{\infty} \delta(x-m N, y-n N) \\
& \otimes \sum_{p=0}^{N-1} \sum_{q=0}^{N-1} \operatorname{rect}(x-p, y-q) \exp \left(i \phi_{p q}\right),
\end{aligned}
$$

where $\delta$ is the Dirac delta function, $\operatorname{rect}(x, y)=$ $\operatorname{rect}(x) \operatorname{rect}(y)$, and

$$
\operatorname{rect}(x)=\left\{\begin{array}{ll}
1 & |x| \leq 0.5 \\
0 & \text { otherwise }
\end{array} .\right.
$$

By the scalar-wave diffraction theorem, the Fraunhofer diffraction can be written as a discrete Fourier transform. ${ }^{37}$ The Fourier series of $t(x, y)$ is

$$
\begin{aligned}
T(m, n)= & \operatorname{sinc}(m / N, n / N) \sum_{p=0}^{N-1} \sum_{1=0}^{N-1} \exp \left(i \phi_{p q}\right) \\
& \times \exp [-i 2 \pi(m p / N+n q / N)],
\end{aligned}
$$

where $\operatorname{sinc}(x, y)=\operatorname{sinc}(x) \operatorname{sinc}(y), \operatorname{sinc}(x)=\sin (\pi x) /$ $\pi x$, and $|T(m, n)|^{2}$ is the intensity of diffraction order $(m, n)$.

Considering the case in which the incident light beams have a flat wave front and a flat intensity level, we can use the mean-square error (MSE) to examine the difference between desired intensity $I(m$, $n)$ and $|T(m, n)|^{2}$. More specifically, as a user tends to worry about only a small number of diffractive orders, the MSE is quite suitable to examine the closeness of experimental and theoretical results of DOE's, where

$$
\mathrm{MSE}=\left.\frac{1}{\#(D)} \sum_{(m, n) \in D}|I(m, n)-\gamma| T(m, n)\right|^{2} \mid,
$$

and $\#(D)$ is the number of elements in $D$. After regression analysis, the linear correlation coefficient $\gamma$ between $I(m, n)$ and $|T(m, n)|^{2}$ is derived to be an index of the correlation and can be explicitly written as

$$
\gamma=\frac{\sum_{(m, n) \in D}\left(I-I_{\text {avg }}\right)\left(|T|^{2}-|T|_{\text {avg }}^{2}\right)}{\left[\sum_{(m, n) \in D}\left(I-I_{\text {avg }}\right)^{2}\right]^{1 / 2}\left[\sum_{(m, n) \in D}\left(|T|^{2}-|T|_{\text {avg }}^{2}\right)^{2}\right]^{1 / 2}},
$$

where $I_{\text {avg }}$ and $|T|_{\text {avg }}^{2}$ are the mean values of $I$ and $|T|^{2}$, respectively.

In fact, when the scalar-wave theorem is used, the whole problem can be simplified to a phase problem $^{37}$ and the amplitude distribution on the image plane can be written as

$$
\begin{aligned}
F\left(k_{x}, k_{y}\right)= & \int_{-\infty}^{\infty} \int_{-\infty}^{\infty} U(x, y) \exp [i \phi(x, y)] \\
& \times \exp \left[-i\left(k_{x} x+k_{y} y\right)\right] \mathrm{d} x \mathrm{~d} y
\end{aligned}
$$

where $k_{x}$ and $k_{y}$ are the wave numbers in the $x$ and the $y$ directions, respectively, $F\left(k_{x}, k_{y}\right)$ is proportional to the diffraction field, and $U(x, y)$ and $\phi(x, y)$ represent light amplitude and phase on the elements, respectively. The main idea behind designing a phase-type DOE is to find the phase and the amplitude distribution solution to each given intensity distribution function $\left|F\left(k_{x}, k_{y}\right)\right|^{2}$. The solutions to these inverse calculation problems, seen in many fields, ${ }^{38}$ are acceptable and comprehensive in some special cases. However, even in cases in which a solution exists, an easier and more efficient calculating method to find such the solution is highly desirable. For a 2-D periodic structure, the solution will not be unique and will depend on detailed information. 39 More specifically, if there are more constraints added, such as a Gaussian distributed light source or a limitation of the feature size during fabrication, mathematical analysis becomes more complicated and the meaning behind the solution more obscure. Now, with our self-repeating units, we can simplify DOE's of interest to the greatest extent in order not to obscure the fundamental meaning behind DOE's.

According to the problem stated above, it appears that a proper heuristic algorithm is required for accomplishing the task. GA's and SA, the two most important heuristic algorithms, are also two of the most commonly used direct search methods in designing a DOE. For the reasons stated below, a GA instead of SA was used in the design of our DOE.

The merit of SA is its ability to search for the best solution among all configurations. Thus the system has opportunities to bypass the local-minimum state and can eventually reside at the global minimum following the Boltzmann distribution. However, SA finds a solution merely according to the predefined energy function and does not consider any information related to real problems known beforehand, i.e., SA can search out a better solution only passively. On the other hand, a GA tends to find a better solution in every generation. More specifically, a GA contains an implicitly parallel processing spirit while evolving within the group. A GA preserves elegant chromosomes from parents to children and improves the quality in advance to reach an optimal consequence. 8,40 Thus we chose a GA to formulate and implement our design, which led to our DOE design as related in Eq. (20).

The aim of our design was to create a unit cell consisting of a $16 \times 16$ element array with phase levels $\pi$ and 0 that can generate an output-energy distribution according to the $7 \times 7$ diffraction image specified in Table 3.

Because our design employs a two-level grating, the transmittance $t(x, y)$ in Eq. (18) is a real number and the intensity distribution generated will be naturally centered symmetrically after the Fourier transform.

\section{A. Parameters and Relevant Strategies}

Referring to the pseudocode of the GA listed in Table 2 , we arbitrarily initiated a population of 32 unit 
Table 3. $7 \times 7$ Diffraction Image Array $^{a}$

\begin{tabular}{rccccccc}
\hline$m / n$ & -3 & -2 & -1 & 0 & 1 & 2 & 0 \\
\hline-3 & 0 & 0 & 0 & 0 & 0 & 0 & 0 \\
-2 & 0 & $C$ & 0 & 0 & 0 & 0 \\
-1 & 0 & 0 & 0 & $A$ & 0 & 0 & 0 \\
0 & 0 & 0 & 0 & 0 & 0 & 0 & 0 \\
1 & 0 & 0 & 0 & 0 & 0 & 0 \\
2 & 0 & $B$ & 0 & 0 & 0 & 0 \\
3 & 0 & 0 & 0 & 0 & 0 & 0 \\
\hline
\end{tabular}

${ }^{a} A=4000 \operatorname{sinc}^{2}(1 / 16,0 / 16), B=8000 \operatorname{sinc}^{2}(2 / 16,2 / 16)$, and $C=6000 \operatorname{sinc}^{2}(2 / 16,2 / 16)$.

cells. These unit cells, which are a set of $16 \times 16$ arrays and are quantified by random phases of 0 or $\pi$, are used as the incipient group. From there, we then started the 2000-generation search from the initial group. The method used to evaluate the fitness within each evolution is the MSE identified in Eq. (21). The output domain $D$ is exactly the central $7 \times$ 7 diffraction image array. Described below are the four methods we applied to identify and obtain the results.

\section{Pure Genetic Algorithm}

The method to generate the subsequent generation and the way the mutations occurred are described below (see Fig. 2). First, the parents were selected and the chromosomes ranked within the group according to fitness in order to decide the opportunity of generating children by a certain probability distribution. The utilized probability distribution $\operatorname{Prob}(i)$ was chosen to be a binomial distribution, i.e., $\operatorname{Prob}(i)$ $=q(1-q)^{i-1}$, where $i$ is a positive integer. At each generation, coupled parents produced a new generation of chromosomes according to its probability. The breeding method was effectively a multipoint method in which every element in the chromosome generated was chosen randomly from among the elements in the same place of the two mating chromosomes. One thing that should be noted is that, because the sum of the finite terms

$$
\begin{aligned}
\sum_{i=1}^{N} q(1-q)^{i-1} & =q\left[1-(1-q)^{N}\right] /[1-(1-q)] \\
& =1-(1-q)^{N}
\end{aligned}
$$

can never equal 1 , a term of probability $(1-q)^{N}$ must be added in. Thus the possibility of mutation was chosen to be $2 /(16 \times 16)$ i.e., $1 / 128$.

\section{Modified Preserving-the-Best Strategy Genetic Algorithm}

With this methodology, the selection of the parents, the production of the children, and the possibility of mutation are all identical to that of the pure GA. However, the best chromosomes will be completely preserved for new generations in this algorithm. In our particular case, only 31 new chromosomes needed to be generated.
3. Modified Preserving-the-Best Strategy Genetic Algorithm with a Random-Search Procedure

This algorithm is almost the same as the modified GA mentioned above, except that a random-search procedure was added. This algorithm not only acquired random terms to generate the new generations, but it also necessitated the addition of some random generating chromosomes to each new generation (Fig. 3). This method corresponds to making the possibility of mutating the second to the twenty-sixth best chromosome to equal $1 / 128$ and the possibility of mutating the last five worst chromosomes to equal 1 (Fig. 2 ). As was stated above, combining the preservingthe-best strategy and increasing the possibility of chromosome mutation into the traditional GA will both guarantee convergence and reduce the possibility of premature locking at the local optimum. The introduction of the random-search procedure agrees well with this understanding. However, the number of random-search chromosomes should be chosen with care, as the modified GA will become a brainless random-search process if all but the best chromosomes are replaced when the random-search process is used. In the example shown, five random initiating chromosomes were added, making a total of only 26 new chromosomes that remained to be passed on from the initial parents.

\section{Modified Preserving-the-Best Strategy Genetic Algorithm Eight-Level Element}

This method is the same as that of Subsection 3.A.2, except that the phase is an eight-level phase i.e., $\{7 \pi / 4,3 \pi / 2,5 \pi / 4, \pi, 3 \pi / 4, \pi / 2, \pi / 4,0\}$.

\section{B. Numerical Results}

The results of all four methods described above are summarized in Fig. 5, in which the $x$ axis is the generation number and the $y$ axis is the merit function that is proportional to the negative value of the MSE.

The numerical values of the diffractive image intensity are shown in Table 4. For comparison with the design goals listed in Table 3 , the linear correlation coefficient [see Eq. (22)] $\gamma=0.9795$. As we can see, $\gamma$ is very close to 1 . This experimental result indicates that the design target has been met. Meanwhile, the design unit cell of the 2-D phase fanout grating is shown at the top of Fig. 6, and the bottom shows the amplitude-type fan-out grating 


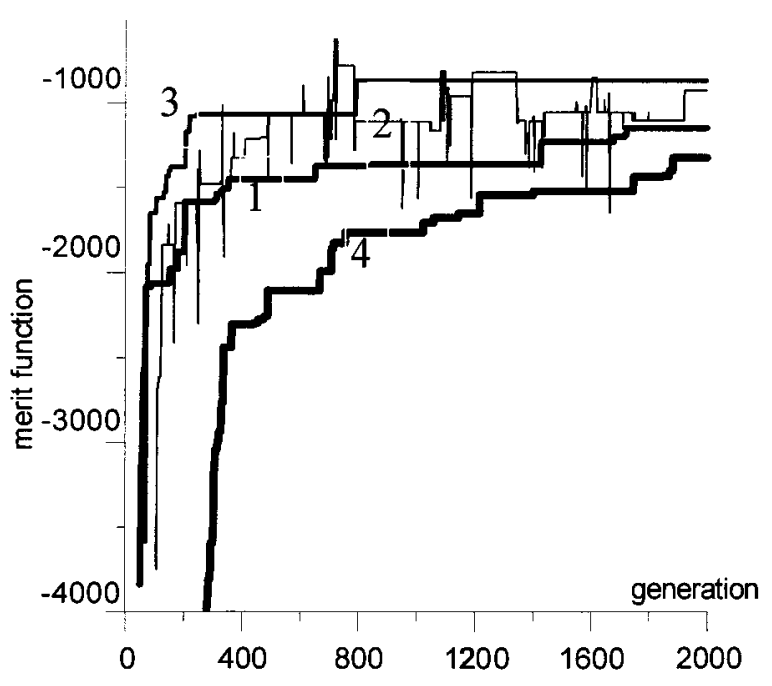

Fig. 5. Numerical results obtained with a GA to design a fan-out grating: (1) modified GA, two-level element; (2) pure GA, twolevel element; (3) modified GA with a random-search procedure: two-level element; (4) modified GA, eight-level element.

that can be transferred to the fabrication process. All calculations were executed on a $133-\mathrm{MHz}$ Pentium CPU PC with Microsoft Windows NT as the operating system. This example produced 2000 generations and took $3704 \mathrm{~s}$ even before the fast Fouriertransform algorithm was adopted to speed up the process. As the Fourier-transform computation was located within four nested loops, the computation speed definitely benefited from the adoption of the fast Fourier transform. It is also clear from Fig. 5 that the modified preserving-the-best strategy GA with a random-search procedure progressed to the final accepted configuration within less than 200 generations, which corresponds to less than $370 \mathrm{~s}$ of computation time. This result is quite acceptable for running the algorithm in a simple Pentium PC environment.

Another example required that we design a $64 \times 64$ pixel grating according to a given $9 \times 9$ diffraction image. We produced 5000 generations in the same way as in the example above and spent 233,617 s to obtain the results. The linear correlation coefficient $\gamma$ was found to be further enhanced to 0.9838 , and this was a marked improvement toward the linear correlation coefficient.
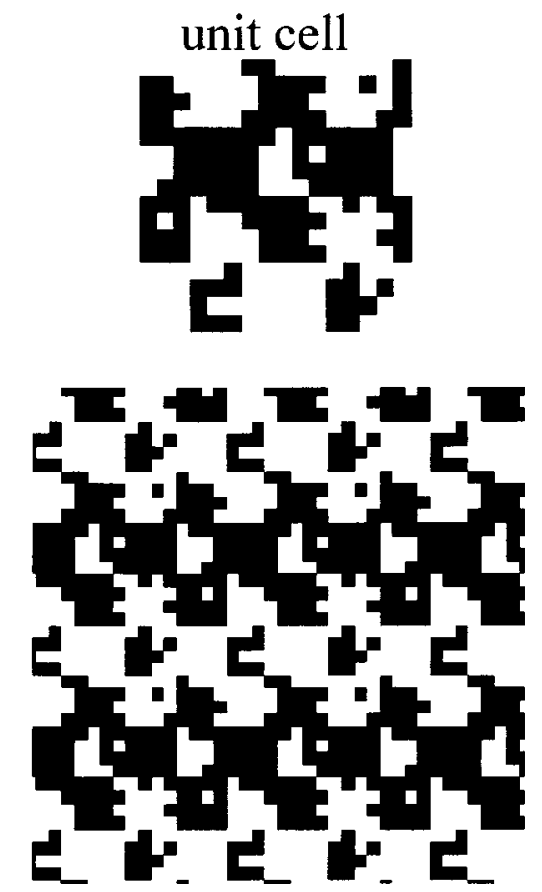

Fig. 6. Fan-out grating designed with a GA.

\section{Fabrication}

The quality of a fabricated DOE is highly influenced by the processing equipment conditions. Furthermore, the design freedom of a DOE is also limited by equipment capabilities, such as feature size. We used a commercially available imagesetter, Agfa SuperSelect 7000 , to produce the binary pattern. Its fine, clear, output feature size was in the range of 30 $\mu \mathrm{m}$. A $10 \times$ photoreduction system was used to generate a reticle to serve as a mask for photolithography. The film was also used directly as a reticle mask. ${ }^{41}$

Our intention in this paper was not to focus on processing accuracy or comparison with products generated from more advanced fabrication facilities. Here only the design concepts are discussed.

\section{A. Photoreduction}

The calculated results of our unit cell were generated by an illustration program from the Aldus Corporation. Aldus FreEHAND uses its tile function to generate repeating patterns of various desired sizes. This

Table 4. Numerical Results of the Diffraction Image Intensity Obtained with a GA

\begin{tabular}{|c|c|c|c|c|c|c|c|}
\hline$m / n$ & -3 & -2 & -1 & 0 & 1 & 2 & 3 \\
\hline-3 & 4.3 & 13.0 & 3.2 & 89.8 & 22.2 & 17.5 & 16.1 \\
\hline-2 & 1.6 & 5392.9 & 26.1 & 13.0 & 13.0 & 7187.5 & 7.7 \\
\hline-1 & 9.0 & 14.5 & 76.1 & 3919.2 & 15.8 & 2.3 & 8.2 \\
\hline 0 & 10.8 & 61.1 & 8.0 & 16.0 & 8.0 & 61.1 & 10.8 \\
\hline 1 & 8.2 & 2.31 & 15.8 & 3919.2 & 76.1 & 14.5 & 9.0 \\
\hline 2 & 7.7 & 7187.5 & 13.0 & 13.0 & 26.1 & 5392.9 & 1.6 \\
\hline 3 & 16.1 & 17.5 & 22.2 & 89.8 & 3.2 & 13.0 & 4.3 \\
\hline
\end{tabular}




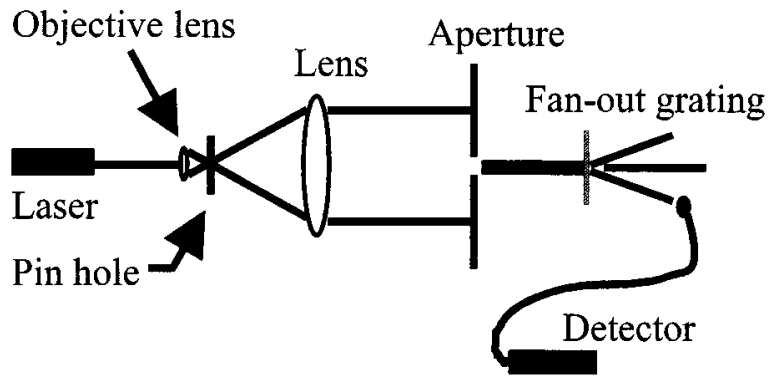

Fig. 7. Layout for diffractive power measurement.

file was transferred to a commercially available imagesetter, Agfa SuperSelect 7000, to produce a film with our desired pattern. ${ }^{9}$ For the purpose of our $10 \times$ photoreduction, a green cold-light illuminating panel made of $\mathrm{ZnS}$ with less than 3\% illumination nonuniformity was used, and a Kodak plate was selected to be the replica.

\section{B. Photolithography}

A Karl Suss g-line direct-contact aligner was used to expose our photoresist (e.g., Fujihunt 6400L) of choice, which was coated onto glass. After many standard development procedures, a pattern constructed by the photoresist was found to have a 0.7 $\mu \mathrm{m}$-deep feature.

Basically an anisotropic dry etch of reactive ion etching can be applied to obtain a precise relief depth. However, to just show only the advantages of the design concept, a transparent binary pattern of photoresist on glass can serve as a phase-type fan-out grating for beam light redistribution.

\section{Measurements}

Diffractive beam light intensity was the object to be measured. The experimental layout (Fig. 7) shows that the laser light was introduced to a spatial filter composed of an object lens and a pinhole. The pinhole position was positioned to the focus of the microscope lens so that the expanded laser light did not move. The collimated light passing through the fanout grating redistributed the beam to the designed pattern, after which a photodetector was used to detect the diffractive light intensity at each spot.

A shear plate ${ }^{42}$ was used to ensure that the collimated light was indeed in parallel. We used a simple interferometer, an optical arrangement in which wedged plates were used to produce a graded path difference between the front and the back surface reflections. Consequently a parallel beam of light produced a linear fringe pattern where the reflections overlapped.

The measured results of the fan-out intensity distribution are shown in Table 5. Values shown at $(0$, 0 ) indicate the zeroth-order term. The linear correlation coefficient $\gamma$ was 0.9775 when the zeroth-order term was neglected. The experimental results show that the intensity of the zeroth-order term deviated from the design value. However, this deviation can be explained by the surface-relief depth. As the program calls for a He-Ne laser, a wavelength of 632.8 $\mathrm{nm}$ was used. The Fujihunt photoresist was found to have a refractive index of $n_{p}=1.64$ at $632.8 \mathrm{~nm}$. The correct etch depth, which corresponds to the halfwavelength difference, should be $0.49 \mu \mathrm{m}[=(632.8$ $\left.\mathrm{nm} / 2) /\left(n_{p}-1\right)\right]$. Our fabricated DOE had an etch depth of $0.7 \mu \mathrm{m}$, which corresponds to a $42 \%$ error. However, despite such a large etch-depth difference variation, only the zeroth-order light distribution was found to have variations.

As the zeroth-order term was neglected, the experimentally measured value of a 0.9795 linear correlation coefficient agrees well with the theoretically predicted linear correlation coefficient of 0.9775 .

In fact, it can be proved that, for a binary element with a phase value of $\{0, \pi\}$, the former denotes region $\mathrm{A}$ and the latter region $\mathrm{B}$. If there is a phase error $\Delta$ generated during fabrication, in general it can be safely assumed that the individual phase errors for each region are $\exp (i \Delta / 2)$ and $\exp [i(\pi-\Delta / 2)]$, respectively.

It is known that the intensity equals the squared term of the field, and the design field for the $k$ th order is defined as follows:

$$
\begin{aligned}
F\left(k_{x}, k_{y}\right)= & \iint_{A} \exp (i 0) \exp \left[-i\left(k_{x}+k_{y}\right)\right] \mathrm{d} x \mathrm{~d} y \\
& +\iint_{B} \exp (i \pi) \exp \left[-i\left(k_{x}+k_{y}\right)\right] \mathrm{d} x \mathrm{~d} y \\
= & \iint_{A} \exp \left(-i\left[k_{x}+k_{y}\right)\right] \mathrm{d} x \mathrm{~d} y \\
& -\iint_{B} \exp \left[-i\left(k_{x}+k_{y}\right)\right] \mathrm{d} x \mathrm{~d} y .
\end{aligned}
$$

\begin{tabular}{|c|c|c|c|c|c|c|c|}
\hline$m / n$ & -3 & -2 & -1 & 0 & 1 & 2 & 3 \\
\hline-3 & 3 & 2 & 10 & 5 & 7 & 10 & 2 \\
\hline-2 & 7 & 1428 & 10 & 10 & 28 & 1957 & 18 \\
\hline-1 & 5 & 7 & 12 & 907 & 20 & 79 & 23 \\
\hline 0 & 2 & 15 & 41 & 9248 & 46 & 20 & 5 \\
\hline 1 & 18 & 78 & 12 & 953 & 30 & 7 & 5 \\
\hline 2 & 15 & 1898 & 12 & 10 & 23 & 1480 & 12 \\
\hline 3 & 2 & 15 & 5 & 5 & 12 & 15 & 18 \\
\hline
\end{tabular}

Table 5. Intensity Distribution of the Designed DOE (in Nanowatts) 
The actual field can be expressed as

$$
\begin{aligned}
\tilde{F}\left(k_{x}, k_{y}\right)= & \iint_{A} \exp (i \Delta / 2) \exp \left[-i\left(k_{x} x+k_{y} y\right)\right] \mathrm{d} x \mathrm{~d} y \\
& +\iint_{B} \exp [i(\pi-\Delta / 2)] \\
& \times \exp \left[-i\left(k_{x} x+k_{y} y\right)\right] \mathrm{d} x \mathrm{~d} y \\
= & \cos (\Delta / 2)\left\{\iint_{A}(1) \exp \left[-i\left(k_{x} x+k_{y} y\right)\right] \mathrm{d} x \mathrm{~d} y\right. \\
& \left.+\iint_{B}(-1) \exp \left[-i\left(k_{x} x+k_{y} y\right)\right] \mathrm{d} x \mathrm{~d} y\right\} \\
& +i \sin (\Delta / 2)\left\{\int \int _ { A } \operatorname { e x p } \left[-i\left(k_{x} x\right.\right.\right. \\
& \left.\left.+k_{y} y\right)\right] \mathrm{d} x \mathrm{~d} y+\iint_{B} \exp \left[-i\left(k_{x} x\right.\right. \\
& \left.\left.\left.+k_{y} y\right)\right] \mathrm{~d} x \mathrm{~d} y\right\} \\
= & \cos (\Delta / 2) F\left(k_{x}, k_{y}\right)+i \sin (\Delta / 2) \delta\left(k_{x}, k_{y}\right),
\end{aligned}
$$

where $\delta$ is the Dirac delta function and

$$
\begin{aligned}
\tilde{F}\left(k_{x}, k_{y}\right)= & \cos (\Delta / 2) F\left(k_{x}, k_{y}\right) \\
& \text { except when } k_{x}=k_{y}=0,
\end{aligned}
$$

that is, for a parallel incident light, the error of a surface-relief depth of a binary DOE will only induce phase error. The surface relief will not influence the intensity ratio of any order except that of the zerothorder term.

\section{Conclusions}

An efficient design methodology for DOE's formed by self-repeating patterns can be generated by a modified preserving-the-best strategy of GA's. Mathematical arguments are provided to show that the preserving-the-best strategy has the sufficient and necessary condition for GA's to converge.

A stochastic process was used to examine the similarity between GA's and SA. The insights generated from this analysis provide us with a way to code these two algorithms in an almost identical manner. Such an approach makes the coding of various heuristic algorithms easy to develop and easy to debug. In addition, the fundamental understanding generated provides us with a way to adjust intelligently the tuning parameters typically found in various heuristic algorithms. Four numerical approaches, the GA's for a binary element, the original GA for a binary element, the modified preserving-the-best GA for a binary element, and the modified preserving- the-best GA for an eight-level element, were used to examine the efficiency of these algorithms. The modified preserving-the-best GA for a binary element was identified to be most efficient in terms of computational time.

Because a simple development platform based on the IBM PC-compatible system was used in the design process, a simple semiconductor process was then adopted to fabricate the DOE's designed. The etching depth was shown not to influence the energy distribution ratio of all nonzero diffractive orders of binary DOE's. This understanding significantly reduces the etching requirement for surface-relief types of DOE's. Experimental results were shown to agree well with theoretical predictions.

This article was partially supported by the National Science Council of Taiwan, China, under project numbers NSC 84-2622-E-002-007 and NSC 85-2622-E-002-017R. The authors thank H. W. Lee for helping us to measure the refractive index of the Fujihunt 6400 photoresist at $632.8 \mathrm{~nm}$. In addition, thanks goes to Julie Lee for her invaluable suggestions and editing to make sure that the accurate meanings were conveyed.

\section{References}

1. J. N. Mait, "Understanding diffractive optic design in the scalar domain," J. Opt. Soc. Am. A 12, 2145-2158 (1995).

2. F. Wyrowski, "Design theory of diffractive elements in the paraxial domain," J. Opt. Soc. Am. A 10, 1553-1561 (1993).

3. M. A. Seldowitz, J. P. Allebach, and D. W. Sweeney, "Synthesis of digital holograms by direct binary search," Appl. Opt. 26, 2788-2798 (1987).

4. J. R. Fienup, "Iterative method applied to image reconstruction and to computer-generated holograms," Opt. Eng. 19, 297-306 (1973).

5. B. K. Jennison, J. P. Allebach, and D. W. Sweeney, "Iterative approaches to computer-generated holography," Opt. Eng. 28, 629-637 (1989).

6. J. Turunen, A. Vasara, and J. Westerholm, "Kinoform phase relief synthesis: a stochastic method," Opt. Eng. 28, 11621167 (1989).

7. M. R. Feldman and C. C. Guest, "High-efficiency hologram encoding for generation of spot arrays," Opt. Lett. 14, 279-481 (1989).

8. D. E. G. Johnson, A. D. Kathman, D. H. Hochmuth, A. L. Cook, D. R. Brown, and B. Delaney, "Advantages of genetic algorithm optimization methods in diffractive optic design," in Diffractive and Miniaturized Optics, S. H. Lee, ed., Vol. CR49 of SPIE Critical Reviews, Bellingham, Wash., 1993), pp. 54-74.

9. R. W. Gerchberg and W. D. Saxton, "A practical algorithm for the determination of phase from image and diffraction plane pictures," Optik 35, 237-246 (1972).

10. N. C. Gallagher and B. Liu, "Method for computing kinoforms that reduces image reconstruction error," Appl. Opt. 12, 23282335 (1973).

11. F. Wyrowski and O. Bryngdahl, "Iterative Fourier-transform algorithm applied to computer holography," J. Opt. Soc. Am. A 5, 1058-1065 (1988).

12. F. Wyrowski, "Diffractive optical elements: iterative calculation of quantized, blazed phase structures," J. Opt. Soc. Am. A 7, 961-969 (1990).

13. F. Wyrowski and O. Bryngdahl, "Digital holography as part of diffractive optics," Rep. Prog. Phys. 54, 1481-1571 (1991).

14. D. E. Goldberg, Genetic Algorithms in Search, Optimization, 
and Machine Learning (Addison-Wesley, Reading, Mass., 1989), Chap. 4, pp. 125-129.

15. J. H. Holland, Adaptation in Natural and Artificial Systems (MIT, Cambridge, Mass., 1992), Chap. 1, pp. 1-19.

16. L. Davis, Genetic Algorithms and Simulated Annealing (Pitman, London, 1987), Chap. 1, pp. 1-11.

17. D. Brown and A. Kathman, "Multi-element diffractive optical designs using evolutionary programming," in Diffractive and Holographic Optics Technology II, Ivan Cindrich and S. H. Lee, eds., Proc. SPIE 2404, 17-27 (1995).

18. S. Kirkpatrick, C. D. Gelatt Jr., and M. P. Vecchi, "Optimization by simulated annealing," Science 220, 671-680 (1983).

19. M. S. Kim and C. C. Guest, "Simulated annealing algorithms for binary phase only filters in pattern classification," Appl. Opt. 29, 1203-1208 (1990).

20. W. H. Press, B. P. Flannery, S. A. Teukolsky, and W. T. Vetterling, Numerical Recipes: The Art of Scientific Recipes (Cambridge U. Press, New York, 1986), Chap. 10, pp. 326-334.

21. D. C. O'Shea, J. W. Beletic, and M. Poutous, "Binary mask generation for diffractive optical elements using microcomputers," in Diffractive Optics: Design, Fabrication, and Applications, Vol. 9 of 1992 OSA Technical Digest Series (Optical Society of America, Washington, D.C., 1992), pp. 114-116.

22. T. J. Suleski and D. C. O'Shea, "Fidelity of PostScriptgenerated masks for diffractive optics fabrication," Appl. Opt. 34, 627-634 (1995).

23. D. C. O'Shea, T. K. Gaylord, J. N. Mait, and A. Kathman, "Course notes," presented at the Diffractive Optics workshop, Georgia Institute of Technology, Atlanta, Georgia, 21-24 March 1995.

24. M. G. Moharam and T. K. Gaylord, "Rigorous coupled-wave analysis of planar-grating diffraction," J. Opt. Soc. Am. 71, 811-818 (1981).

25. T. K. Gaylord and M. G. Moharam, "Analysis and application of optical diffraction gratings," Proc. IEEE 73, 894-937 (1985).

26. M. G. Moharam and T. K. Gaylord, "Rigorous coupled-wave analysis of metallic surface-relief gratings," J. Opt. Soc. Am. A 3, 1780-1788 (1986).

27. E. N. Glytsis and T. K. Gaylord, "Rigorous three-dimensional coupled-wave diffraction analysis of single and cascaded anisotropic gratings," J. Opt. Soc. Am. A 4, 2061-2080 (1987).
28. R. Magnusson and T. K. Gaylord, "Equivalence of multiwave coupled-wave theory and modal theory of periodic-media diffraction,” J. Opt. Soc. Am. 68, 1777-1779 (1978).

29. M. G. Moharam and T. K. Gaylord, "Formulation for stable and efficient implementation of rigorous coupled-wave analysis of binary gratings," J. Opt. Soc. Am. A 12, 1068-1076 (1995).

30. G. Granet and B. Guizal, "Efficient implementation of the coupled-wave method for metallic lamellar gratings in TM polarization,” J. Opt. Soc. Am. A 13, 1019-1023 (1996).

31. E. Johnson, M. A. G. Abushagar, and A. Kathman, "Phase grating optimization using genetic algorithms," in Optical Design for Photonics, Vol. 9 of 1993 OSA Technical Digest Series (Optical Society of America, Washington, D.C., 1993), pp. 7173.

32. E. Aarts and J. Korst, Simulated Annealing and Boltzmann machines, (Wiley, New York, 1989), Chap. 2, pp. 13-14.

33. J. H. Holland, Adaptation in Natural and Artificial Systems, (MIT, Cambridge, Mass., 1992), Chap. 3, pp. 49-52.

34. Ref. 32, Chap. 3, pp. 49-52.

35. S. M. Ross, Stochastic Process (Wiley, New York, 1983), pp. $100-111$.

36. D. Geman, "Random fields and inverse problems in imaging," in Proceedings of the École d'Été de Probabilités de Saint-Flour XVIII-1988, Lecture Notes in Mathematics, Vol. 1427, (Springer-Verlag, New York, 1991), pp. 113-193.

37. J. W. Goodman, Introduction to Fourier Optics, 1st ed. (McGraw-Hill, New York, 1992), Chap. 4, pp. 57-70.

38. M. Nieto-Vesperinas, Scattering and Diffraction in Physical Optics (Wiley, New York, 1991), Chap. 10, pp. 341-376.

39. P. Millane, "Phase problems for periodic images: effects of support and symmetry," J. Opt. Soc. Am. A 10, 1037-1045 (1993).

40. Ref. 14, Chap. 2, pp. 27-57.

41. P. S. Levin and L. H. Domash, "MacBeep: a desktop system for binary optics," in Diffractive Optics: Design, Fabrication, and Applications, Vol. 9 of 1992 OSA Technical Digest Series (Optical Society of America, Washington, D.C., 1992), pp. 120 122.

42. "Lasers and instruments guide," B.5, 12-16, Melles Griot Corp., 4665 Nautilus Court, South Boulder, Colorado (1994). 